\title{
Clinical and Polysomnographic Characteristics of Patients with Restless Legs Syndrome
}

\author{
James Kilsung Yang, MHS, Moonsook Lee, MD \\ Busan Sleep Center, Busan, Korea
}

\author{
Received: April 12, 2019 \\ Accepted: June 21, 2019 \\ Correspondence \\ James Kilsung Yang, MHS \\ Busan Sleep Center, 708 Jungang-daero, \\ Busanjin-gu, Busan 47291, Korea \\ Tel +82-51-818-2526 \\ Fax +82-51-818-2527 \\ E-mail jameskilsung@naver.com \\ ORCID \\ James Kilsung Yang \\ https://orcid.org/0000-0001-7633-9471 \\ Moonsook Lee \\ https://orcid.org/0000-0002-5895-2114
}

Background and Objective Although restless legs syndrome (RLS) symptoms are relatively clear, many people are suffering from RLS without proper care because RLS is often underdiagnosed and misdiagnosed. Purpose of this study was to examine clinical and polysomnographic aspects of RLS to better understand the disorder for future diagnosis.

Methods This study enrolled 113 idiopathic untreated RLS patients, including 74 (65.5\%) women and 39 (34.5\%) men. Restless legs syndrome rating scale (RLSRS) and RLS checklist were examined. All patients underwent suggested immobilization test (SIT) and polysomnogram (PSG).

Results RLS occurred in all age groups. It was the most prevalent in age groups of 50s and 60s. On average, RLS was diagnosed 12.5 years after the first occurrence of RLS symptoms. RLS occurred twice as often among women than that among men. Based on average RLSRS, 57.5\% and $26.5 \%$ of patients belonged to severe and very severe categories, respectively. While symptoms were the most prominent between $8 \mathrm{PM}$ and $4 \mathrm{AM}$, patients reported that calves were the main site of discomfort. Of all participants, $54.9 \%$ reported family history of RLS. RLS patients' PSGs showed lower sleep efficiency (SE), total sleep time, and slow wave percentage with higher leg movement arousal index (LMAI), arousal index, and sleep latency (SL). RLSRS was positively correlated with LMAI $(\mathrm{r}=0.221, \mathrm{p}<0.05)$, periodic leg movement during wake index-PSG (PLMWI-PSG) $(\mathrm{r}=$ $0.214, \mathrm{p}<0.05)$, and SL $(\mathrm{r}=0.207, \mathrm{p}<0.05)$. RLSRS was also negatively correlated with SE $(\mathrm{r}=$ $-0.222, \mathrm{p}<0.05)$. RLSRS was not correlated with PLMWI-SIT or PLMI.

Conclusions The results suggest that the severity of RLS patients is related to SL, LMAI, PLMWI-PSG, and SE. In order to effectively diagnose RLS, not only clinical characteristics, but also polygraphic characteristics such as SL, LMAI, PLMWI-PSG, and SE should be taken into account.

Sleep Med Res 2019;10(1):43-48

Key Words Restless legs syndrome, Characteristics, Correlates, Polysomnography.

\section{INTRODUCTION}

Restless legs syndrome (RLS) is a debilitating neurologic disorder characterized by unpleasant, deep-seated paresthesias in legs and sometimes in arms during periods of rest or inactivity. The International Restless Legs Syndrome Study Group (IRLSSG) has described the following four essential criteria to establish the diagnosis of idiopathic RLS [1]: 1) an urge to move legs, usually accompanied or caused by uncomfortable and unpleasant sensations in legs; 2) the urge to move or unpleasant sensations begin or worsen during periods of rest or inactivity such as lying or sitting; 3 ) the urge or unpleasant sensations are partially or totally relieved by movement such as walking or stretching, at least as long as the activity continues; and 4) the urge or unpleasant sensations are worse in the evening or nighttime than that during the day or only occur in the evening or night.

The prevalence of RLS is around 5-10\% in the Western countries [2-6]. There might be some racial differences regarding the prevalence of RLS as it is about $0.1-3.2 \%$ lower in Asian coun- 
tries [7-10]. RLS is strongly associated with not only insomnia [11], but also psychiatric conditions [12], decreased quality of life [13], and high mortality rate [14]. Although RLS symptoms are relatively clear, many people are suffering from RLS without proper treatment because it is often underdiagnosed and misdiagnosed. Therefore, a correct diagnosis and an appropriate management are important. The objective of this study was to examine clinical and polysomnographic features of RLS to better understand the disorder for future diagnosis.

\section{METHODS}

This study enrolled 113 idiopathic untreated Korean RLS patients, including 74 women (65.5\%) and 39 men (34.5\%) with chief complaints of RLS symptoms among 150 patients who visited our primary sleep center from May 2016 to April 2017. Thirty-seven patients were excluded due to possible secondary RLS or refusal to perform polysomnogram (PSG) and suggested immobilization test (SIT) for diagnosis. All subjects enrolled in this study were diagnosed with RLS according to the criteria of the IRLSSG [1]. Restless legs syndrome rating scale (RLSRS) [1] and RLS checklist were also examined. RLSRS included 10 questions such as the severity and frequency of RLS symptoms and other disabilities caused by RLS. Each question was rated with a five-point scale from 0 to 4, with higher RLSRS score indicating more severe case. RLSRS is the most widely used rating scale to evaluate the severity of RLS symptoms [15]. It has been validated in a large multi-centered study, showing adequate reliability, internal consistency, and criterion validity [1]. RLSRS was translated into Korean language by a bilingual first author in this study. RLS checklist included age, gender, onset year of RLS, frequency of RLS, most frequent sites of RLS, worst time of RLS, family history, and so on. A family history was defined as having at least one first-degree relative with the RLS phenotype according to the criteria of the IRLSSG. Family history was investigated by a face-to-face interview with patients mostly, or with patient and family member(s).

All patients went through SIT and PSG using standard method. The SIT was a 60 minutes test. It was performed one hour prior to the usual bedtime [16]. During SIT, the patients sat on a bed reclined at 45 degrees with their legs stretched out. They were instructed to limit their movements and stay awake during the test. After SIT was completed, PSG was performed.

Sleep studies were recorded using Somnologica Studio 3.332. Four EEG derivations (C4/A1, C3/A2, C4/O1, C3/O2), two EOG, submentalis EMG, EKG, separate right and left anterior tibialis EMG, and body position were recorded. In addition, readings from one nasal-oral thermistor, one nasal pressure monitor, a snore microphone, chest and abdominal respiratory effort belts, and a finger pulse oximeter were recorded. Electromyogram amplifiers were set at a sensitivity of 1 with high fil- ters at $30 \mathrm{~Hz}$ and low filters at $0.1 \mathrm{~Hz}$.

Sleep records were scored visually according to standard criteria [17] by trained technicians. Apnea was identified if airflow was absent or nearly absent $\geq 10 \mathrm{sec}$. Hypopnea was considered if there was $\geq 30 \%$ reduction in airflow for $\geq 10 \mathrm{sec}$ with a $4 \%$ decrease in oxygen saturation. The apnea-hypopnea index was defined as the number of apneas or hypopneas per hour of sleep.

A leg movement (LM) was defined as a burst of anterior tibialis muscle activity with duration of $0.5-5$ seconds between onset and resolution having an amplitude of at least 25\% higher than the baseline during biocalibration. However, this study used 10 seconds as the upper limit for SIT because a large number of movements during wakefulness exceeded 5 seconds [18]. Periodic leg movements (PLM) were defined as LM in one or both anterior tibialis muscles occurring in a series of four such events with intermovement intervals between 5 and 90 seconds, consistent with ASDA criteria [19]. PLM indices during waking and sleep [periodic leg movements waking index (PLMWI) and periodic leg movements index (PLMI), respectively] were derived by dividing the total number of PLM by the total waking and sleep time (in hours), respectively.

The presence of medical conditions known to be associated with RLS was ruled out by clinical evaluation and blood tests including serum iron, total iron binding capacity, ferritin, BUN, and creatinine. Patients with ferritin levels less than $50 \mathrm{ng} / \mathrm{mL}$ were excluded from this study because these patients might have secondary RLS. Patients taking benzodiazepines, antidepressants, antipsychotics, opiates, and dopaminergic medications for at least one month prior to PSG evaluations were also excluded. This study was approved by the local Institutional Ethics Committee. A written informed consent was obtained from each patient.

Data were explored using descriptive methods to calculate means, standard deviations, and ranges. Differences between gender were calculated using t-test with a significance level of 0.05 . Spearman's rank correlation coefficient was used to examine statistical correlation between polysomnographic variables. Scatter plots of RLSRS and polysomnographic variables were created to visually examine correlation. All statistical analyses were conducted using SAS (SAS version 9.3, SAS Institute, Cary, NC, USA).

\section{RESULTS}

\section{Demographic and Clinical Results}

Clinical characteristics of subjects are shown in Table 1. Mean age of the study sample was $48.0 \pm 16.8$ years (range, $7-78$ years). Age distribution is shown in Fig. 1. Majority (54.9\%) of subjects were in their 50s and 60s. Regarding RLS severity, 2 (1.8\%) patients in the mild category, $16(14.2 \%)$ in the moderate category, $65(57.5 \%)$ in the severe category, and $30(26.5 \%)$ in the very se- 
vere category were found. Mean frequency of RLS was 6.2 days per week. Eighty-nine (78.8\%) patients had RLS 7 days per week.

Symptoms of RLS were most prevalent between 8 PM and 4 AM (Fig. 2). Patients' symptom complaints were mostly be-

Table 1. Main demographic, clinical, and polysomnographic characteristics of subjects

\begin{tabular}{lcc}
\hline & Mean \pm SD & Range \\
\hline Gender & 74 women $(65.5 \%)$ / 39 men $(34.5 \%)$ \\
Age (yrs) & $48.0 \pm 16.8$ & $7-78$ \\
Age of onset (yrs) & $35.3 \pm 16.2$ & $2-68$ \\
Duration of illness (yrs) & $12.5 \pm 11.4$ & $0.2-50$ \\
RLSRS score & $26.4 \pm 6.2$ & $8-38$ \\
Frequency of RLS (/wk) & $6.2 \pm 0.6$ & $1-7$ \\
BMI (kg/m ${ }^{2}$ ) & $22.9 \pm 3.1$ & $12.0-32.4$ \\
PLMWI-SIT & $56.2 \pm 48.9$ & $0-204.0$ \\
PLMWI-PSG & $39.5 \pm 37.0$ & $0-214.4$ \\
PLMI & $21.0 \pm 30.9$ & $0-181.1$ \\
AI & $20.3 \pm 12.6$ & $3.6-94.8$ \\
LMAI & $6.2 \pm 11.0$ & $0-92.2$ \\
TST (min) & $344.8 \pm 57.6$ & $203.0-458.5$ \\
Sleep latency (min) & $14.0 \pm 19.4$ & $0.5-122.0$ \\
Sleep efficiency (\%) & $82.2 \pm 12.0$ & $47.1-99.3$ \\
Stage N1 (min) & $15.0 \pm 9.5$ & $1.7-51.0$ \\
Stage N2 (min) & $53.6 \pm 11.0$ & $25.9-89.7$ \\
Stage N3 (min) & $12.6 \pm 11.8$ & $0-50.2$ \\
Stage R (min) & $19.2 \pm 6.4$ & $3.8-38.7$ \\
\hline RLS: resles & &
\end{tabular}

RLSRS: restless legs syndrome rating scale, RLS: restless legs syndrome, BMI: body mass index, PLMWI-SIT: periodic leg movement waking index in suggested immobilization test, PLMWIPSG: periodic leg movement waking index in polysomnogram, PLMI: periodic leg movement index, AI: arousal index; LMAI: leg movement arousal index, TST: total sleep time. tween 9 PM and 1 AM. Among patients' various RLS symptoms (Fig. 3), tingling sensation and numbness were the most common ones. The most distressed parts of the body were in the order of calves, thighs, and shins (Fig. 4). Symptoms of RLS in the upper limb occurred in 13\% of subjects. Of 113 patients, 62 (54.9\%) showed family history. Eighty-eight (77.9\%) patients had daytime symptoms, although the degree was milder. Routine blood tests (including serum iron, TIBC, ferritin, BUN, creatinine) were normal. Mean ferritin levels of subjects were 117.5

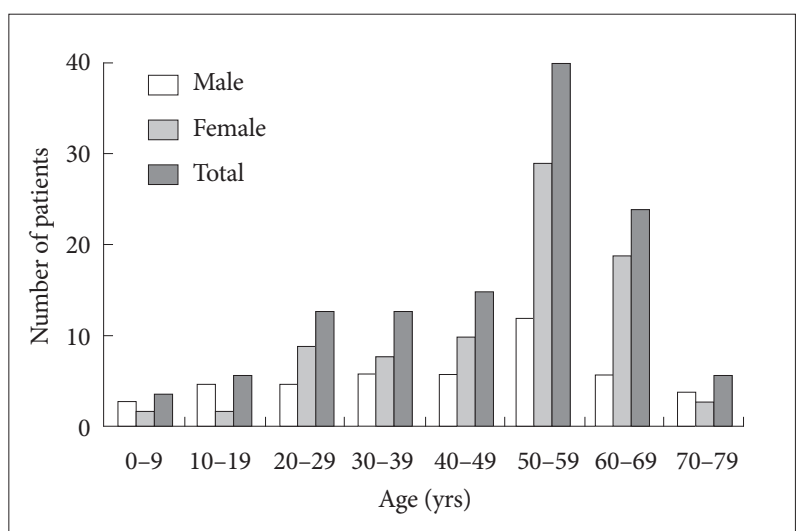

Fig. 1. Age distribution.

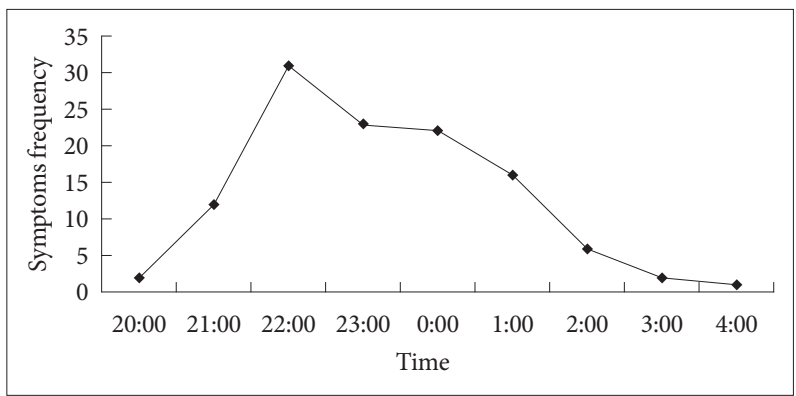

Fig. 2. Most prevalent time of restless legs syndrome symptoms.

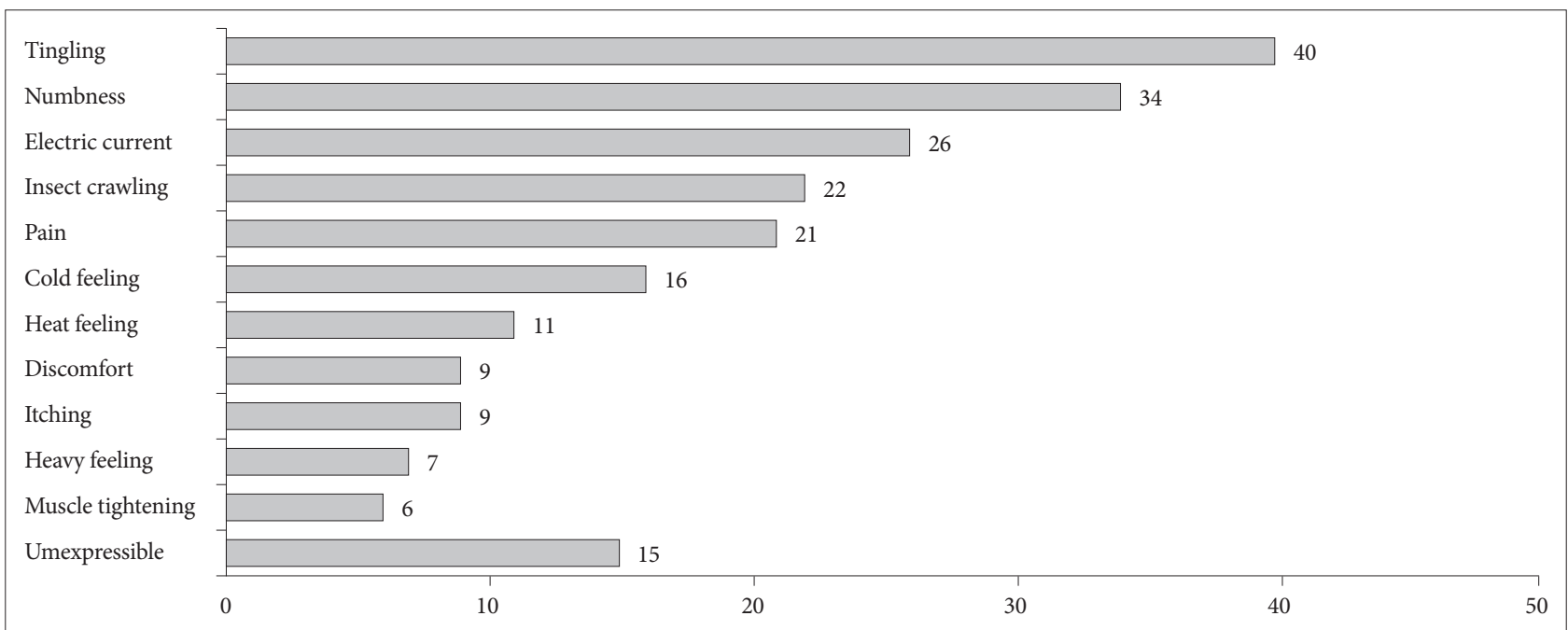

Fig. 3. Symptoms described by patients. The numbers represent multiple responses of restless legs syndrome symptoms from each patient. 
$\pm 75.0 \mathrm{ng} / \mathrm{mL}$.

\section{Polysomnographic Results}

Polysomnographic results are shown in Table 1. Using PLMS diagnosis criteria of PLMI > 15, PLMI > 10, and PLMI > 5, $37.2 \%, 46 \%$, and $60.2 \%$ of subjects were diagnosed with PLMS, respectively. When using PLMWI $>40$ and PLMWI $>30$ during PSG, $42.9 \%$ and $60.2 \%$ of patients were diagnosed with RLS, respectively. If the same PLMWI criteria were used in a SIT, $53.6 \%$ and $58.9 \%$ of patients were diagnosed with RLS, respectively. There were no significant differences in PSG variables between men and women.

\section{Correlation Results}

Correlations between polysomnographic variables are depicted in Table 2. RLSRS did not show any correlation with serum iron, TIBC, or ferritin. RLSRS was positively correlated with leg movement arousal index (LMAI) $(r=0.221, p<0.05)$ (Fig. 5), PLMWI-PSG $(r=0.214, p<0.05)$ (Fig. 6), and sleep latency (SL) $(\mathrm{r}=0.207, \mathrm{p}<0.05)$. Also, RLSRS was negatively correlated with sleep efficiency $(\mathrm{SE})(\mathrm{r}=-0.222, \mathrm{p}<0.05)$ (Fig. 7). However,

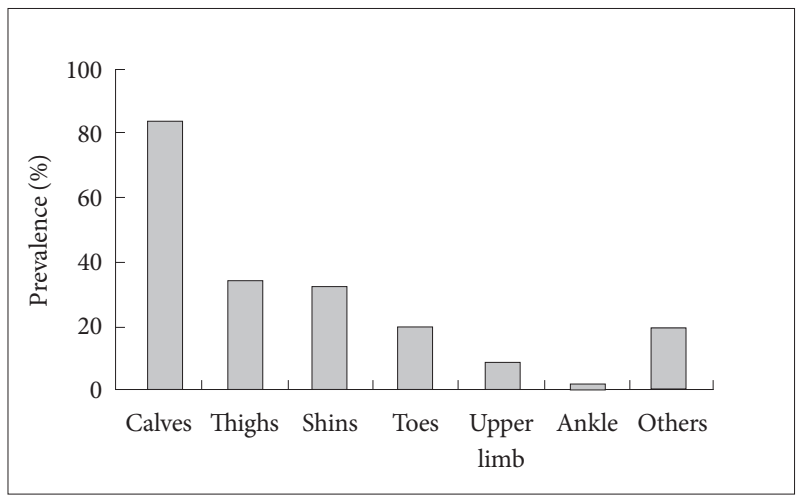

Fig. 4. Most prevalent sites of distress.
RLSRS did not show any correlation with arousal index (AI), PLMWI-SIT, PLMI, or total sleep time (TST). Moreover, AI was positively correlated with LMAI $(\mathrm{r}=0.358, \mathrm{p}<0.001)$ and PLMI ( $\mathrm{r}=0.274, \mathrm{p}<0.05)$. On the other hand, TST $(\mathrm{r}=-0.317$, $\mathrm{p}<0.05)$ and SE $(\mathrm{r}=-0.301, \mathrm{p}<0.002)$ had a negative correlation with AI. PLMWI-SIT was positively correlated with LMAI $(\mathrm{r}=0.228, \mathrm{p}<0.05)$ and PLMWI-PSG $(\mathrm{r}=0.219, \mathrm{p}<0.05)$. Also, SL was positively correlated with PLMI $(\mathrm{r}=0.195, \mathrm{p}<0.05)$ and PLMWI-PSG $(\mathrm{r}=0.379, \mathrm{p}<0.001)$. PLMWI-PSG was positively correlated with LMAI $(\mathrm{r}=0.456, \mathrm{p}<0.001)$.

\section{DISCUSSION}

Results of this study demonstrated many polysomnographic and clinical characteristics of RLS. These subjects had a wide range of age (7-78 years old), indicating that people at all ages could develop RLS. The average age of RLS patients was 48 years old. About half (54.9\%) of subjects were in age groups of

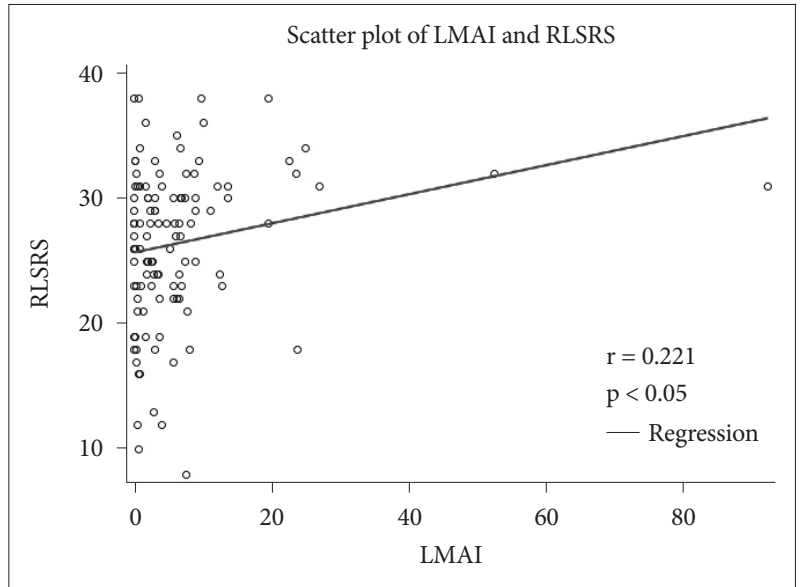

Fig. 5. Correlation between leg movement arousal index (LMAI) and restless legs syndrome rating scale (RLSRS).

Table 2. Correlations between RLSRS and polysomnographic variables of restless legs syndrome

\begin{tabular}{|c|c|c|c|c|c|c|c|c|c|}
\hline & RLSRS & AI & LMAI & PLMWI-SIT & PLMWI-PSG & PLMI & SL & TST & SE \\
\hline RLSRS & 1 & 0.116 & $0.221^{*}$ & -0.028 & $0.214^{*}$ & 0.132 & $0.207^{*}$ & -0.163 & $-0.222 *$ \\
\hline $\mathrm{AI}$ & & 1 & $0.358^{\dagger}$ & -0.148 & 0.099 & $0.274^{*}$ & 0.178 & $-0.317^{*}$ & $-0.301^{*}$ \\
\hline LMAI & & & 1 & 0.228 & $0.456^{\dagger}$ & $0.717^{\dagger}$ & $0.187^{*}$ & $-0.253^{*}$ & $-0.308^{*}$ \\
\hline PLMWI-SIT & & & & 1 & $0.219^{*}$ & 0.158 & -0.158 & -0.039 & -0.022 \\
\hline PLMWI-PSG & & & & & 1 & $0.323^{*}$ & $0.379^{\dagger}$ & $-0.453^{\dagger}$ & $-0.500^{\dagger}$ \\
\hline PLMI & & & & & & 1 & $0.195^{*}$ & -0.182 & $-0.254^{*}$ \\
\hline SL & & & & & & & 1 & -0.408 & $-0.436^{\dagger}$ \\
\hline TST & & & & & & & & 1 & $0.824^{\dagger}$ \\
\hline SE & & & & & & & & & 1 \\
\hline
\end{tabular}

${ }^{*} \mathrm{p}<0.05 .{ }^{\dagger} \mathrm{p}<0.001$.

RLSRS: restless legs syndrome rating scale, AI: arousal index, LMAI: leg movement arousal index, PLMWI-SIT: periodic leg movement waking index in suggested immobilization test, PLMWI-PSG: periodic leg movement waking index in polysomnogram, PLMI: periodic leg movement index, TST: total sleep time, SL: sleep latency, SE: sleep efficiency. 


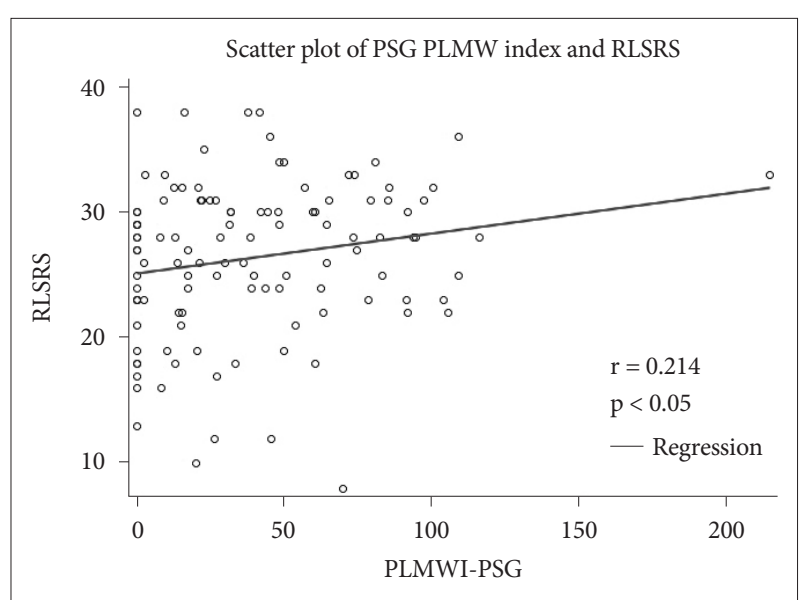

Fig. 6. Correlation bewteen periodic leg movement waking index in polysomnogram (PLMWI-PSG) and restless legs syndrome rating scale (RLSRS).

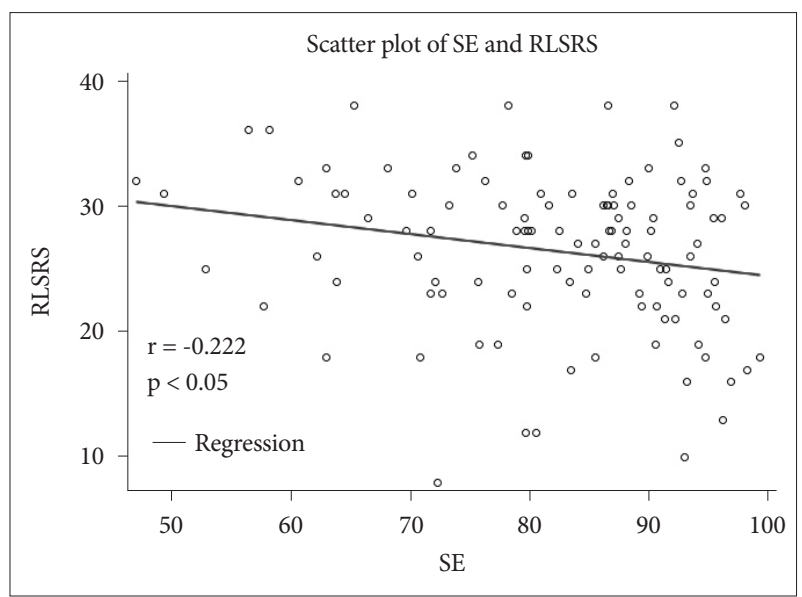

Fig. 7. Correlation between sleep efficiency (SE) and restless legs syndrome rating scale (RLSRS).

50s and 60s. RLS occurred twice as often in women than in men, consistent with previous studies [10,20,21]. Although the average onset age of RLS was 35.3 years, a significant problem was the late diagnosis with patients being identified with RLS 12.5 years after the onset. Even with rather simple diagnosis criteria for RLS, reasons for such late diagnosis could be due to various patient descriptions and possibly a lack of understanding of RLS as a medically significant disorder. In addition, RLS requires a differential diagnosis from many disorders such as neuropathic pain syndrome, arthritis, leg cramps, positional discomfort, restless insomnia, painful legs and moving toes, druginduced akathisia, and so on.

Subjects were categorized as having severe cases of RLS. They sought professional care in a sleep clinic to relieve their RLS symptoms. Furthermore, $13 \%$ of patients reported experiencing symptoms of RLS in the upper body and $77.9 \%$ reported experiencing symptoms during day time. Considering that previous studies have found more severe RLS cases with having previously mentioned symptoms [22,23], our results indicate that most patients already have severe RLS. Several studies have reported 54.9-63\% of patients have family history of RLS [24,25]. Similarly, the present study found that $54.9 \%$ of patients reported family history of RLS. Therefore, when diagnosing RLS, family history of both children and adults is important.

RLS symptoms vary according to circadian rhythm [26]. The present study revealed that patients had the highest severity of RLS between 9 PM and 1 AM. Because people usually fall asleep during this period, RLS can be a crucial reason for insomnia. More than $90 \%$ of RLS patients reported difficulties in initiating or maintaining sleep [24,27]. Some PLMs cause arousal during sleep. However, this study found a positive correlation between RLSRS and LMAI, but no relation between RLSRS and AI or PLMI. Therefore, we concluded that the severity of RLS was more correlated with LMAI than with AI or PLMI. RLSRS also showed positive correlation with LMAI, PLMWI-PSG, and SL, although it showed a negative correlation with SE. Therefore, we believe that the severity of RLS patients is related to longer SL, higher LMAI, higher PLMWI-PSG, and poorer SE.

PLMI and PLMW during PSG and SIT, respectively, were markers for RLS severity in the literature [28,29]. However, our study showed a low RLS diagnostic rate using PLMI and PLMWI-SIT. Only $60.2 \%$ of subjects had PLMI $>5$ in PSG. Elevated PLM indices have been reported for patients with narcolepsy [30], obstructive sleep apnea [31], REM-sleep behavior disorder [32], Parkinson's disease [33], and healthy subjects [34]. In addition, PLMI has a high night-to-night variation [35]. SIT also showed a significant test-to-test variability [36] such as when the test was performed, it was hard to control important subjective influences (e.g., distraction and attention level). Hence, the use of just polygraphic characteristics, PLMI, and PLMWI-SIT to diagnose RLS is insufficient.

The first limitation of our study was that the majority (84\%) of patients from a sleep clinic had severe or very severe cases of RLS. Therefore, it was hard to extrapolate results and apply them to a larger RLS population. Secondly, there was a possibility of first night effect for patients because polygraphic recordings were done just once in a laboratory environment. In addition, this study did not have healthy subjects as control for comparison.

In summary, results of this study demonstrated many clinical and polysomnographic characteristics of RLS. RLS most commonly occurred in age groups of 50s and 60s, although it occurred in all ages. RLS was twice as prevalent in women as that in men. RLS symptoms occurred mostly from 8 PM to 4 AM with the calf being the main site of discomfort. About half (54.9\%) subjects reported family history of RLS. SL, LMAI, PLMWI-PSG, and SE were all related to the severity of RLS. Finally, in order to effectively diagnose RLS, not only clinical characteristics, but also polygraphic characteristics such as SL, LMAI, PLMWI-PSG, and SE should be taken into account. 


\section{Conflicts of Interest}

The authors have no financial conflicts of interest.

\section{Authors' Contribution}

Conceptualization: Yang JK, Lee M. Data curation: Yang JK, Lee M. Formal analysis: Yang JK, Lee M. Methodology: Yang JK, Lee M. Project administration: Yang JK. Resources: Yang JK, Lee M. Software: Yang JK, Lee M. Supervision: Yang JK. Validation: Yang JK, Lee M. Visualization: Yang JK, Lee M. Writing_original draft: Yang JK. Writing_review \& editing: Yang JK, LeeM.

\section{REFERENCES}

1. Allen RP, Kushida CA, Atkinson MJ; RLS QoL Consortium. Factor analysis of the International Restless Legs Syndrome Study Group's scale for restless legs severity. Sleep Med 2003;4:133-5.

2. Allen RP, Dean T, Earley CJ. Effects of rest-duration, time-of-day and their interaction on periodic leg movements while awake in restless legs syndrome. Sleep Med 2005;6:429-34.

3. Berger K, Luedemann J, Trenkwalder C, John U, Kessler C. Sex and the risk of restless legs syndrome in the general population. Arch Intern Med 2004;164:196-202.

4. Ulfberg J, Nyström B, Carter N, Edling C. Prevalence of restless legs syndrome among men aged 18 to 64 years: an association with somatic disease and neuropsychiatric symptoms. Mov Disord 2001;16:1159-63.

5. Tison F, Crochard A, Léger D, Bouée S, Lainey E, El Hasnaoui A. Epidemiology of restless legs syndrome in French adults: a nationwide survey: the INSTANT Study. Neurology 2005;65:239-46.

6. Phillips B, Hening W, Britz P, Mannino D. Prevalence and correlates of restless legs syndrome: results from the 2005 National Sleep Foundation Poll. Chest 2006;129:76-80.

7. Tan EK, Seah A, See SJ, Lim E, Wong MC, Koh KK. Restless legs syndrome in an Asian population: a study in Singapore. Mov Disord 2001; 16:577-9.

8. Sevim S, Dogu O, Camdeviren H, Bugdayci R, Sasmaz T, Kaleagasi H, et al. Unexpectedly low prevalence and unusual characteristics of RLS in Mersin, Turkey. Neurology 2003;61:1562-9.

9. Mizuno S, Miyaoka T, Inagaki T, Horiguchi J. Prevalence of restless legs syndrome in non-institutionalized Japanese elderly. Psychiatry Clin Neurosci 2005;59:461-5.

10. Cho SJ, Hong JP, Hahm BJ, Jeon HJ, Chang SM, Cho MJ, et al. Restless legs syndrome in a community sample of Korean adults: prevalence, impact on quality of life, and association with DSM-IV psychiatric disorders. Sleep 2009;32:1069-76.

11. Budhiraja P, Budhiraja R, Goodwin JL, Allen RP, Newman AB, Koo $\mathrm{BB}$, et al. Incidence of restless legs syndrome and its correlates. J Clin Sleep Med 2012;8:119-24.

12. Szentkiralyi A, Völzke H, Hoffmann W, Baune BT, Berger K. The relationship between depressive symptoms and restless legs syndrome in two prospective cohort studies. Psychosom Med 2013;75:359-65.

13. Cho YW, Kim DH, Allen RP, Earley CJ. Assessing health-related quality of life in patients with restless legs syndrome in Korea: comparison with other chronic medical diseases. Sleep Med 2012;13:1158-63.

14. Li Y, Wang W, Winkelman JW, Malhotra A, Ma J, Gao X. Prospective study of restless legs syndrome and mortality among men. Neurology 2013;81:52-9.

15. Garcia-Borreguero D, Larrosa O, de la Llave Y, Granizo JJ, Allen R. Correlation between rating scales and sleep laboratory measurements in restless legs syndrome. Sleep Med 2004;5:561-5.

16. Montplaisir J, Boucher S, Nicolas A, Lesperance P, Gosselin A, Rompré
P, et al. Immobilization tests and periodic leg movements in sleep for the diagnosis of restless leg syndrome. Mov Disord 1998;13:324-9.

17. Rechtschaffen A, Kales A. A manual of standardized terminology, techniques and scoring system for sleep stages of human subjects. Bethesda (MD): US National Institute of Neurological Diseases and Blindness, Neurological Information Network 1968.

18. Michaud M, Poirier G, Lavigne G, Montplaisir J. Restless Legs Syndrome: scoring criteria for leg movements recorded during the suggested immobilization test. Sleep Med 2001;2:317-21.

19. The Atlas Task Force. Recording and scoring leg movements. Sleep 1993; 16:749-59.

20. Rothdach AJ, Trenkwalder C, Haberstock J, Keil U, Berger K. Prevalence and risk factors of RLS in an elderly population: the MEMO study. Neurology 2000;54:1064-8.

21. Garcia-Borreguero D, Egatz R, Winkelmann J, Berger K. Epidemiology of restless legs syndrome: the current status. Sleep Med Rev 2006;10: $153-67$.

22. Chaudhuri KR. Augmentation and rebound. In: Chaudhuri KR. Odin P, Olanow CW. Restless kegs syndrome. Abingdon: Taylor \& Francis 2004;109-11.

23. Michaud M, Chabli A, Lavigne G, Montplaisir J. Arm restlessness in patients with restless legs syndrome. Mov Disord 2000;15:289-93.

24. Montplaisir J, Boucher S, Poirier G, Lavigne G, Lapierre O, Lespérance P. Clinical, polysomnographic, and genetic characteristics of restless legs syndrome: a study of 133 patients diagnosed with new standard criteria. Mov Disord 1997;12:61-5.

25. Winkelmann J, Wetter TC, Collado-Seidel V, Gasser T, Dichgans M, Yassouridis A, et al. Clinical characteristics and frequency of the hereditary restless legs syndrome in a population of 300 patients. Sleep 2000; 23:597-602.

26. Baier PC, Trenkwalder C. Circadian variation in restless legs syndrome. Sleep Med 2007;8:645-50.

27. Hornyak M, Feige B, Voderholzer U, Philipsen A, Riemann D. Polysomnography findings in patients with restless legs syndrome and in healthy controls: a comparative observational study. Sleep 2007;30:861-5.

28. Trenkwalder C, Walters AS, Hening W. Periodic limb movements and restless legs syndrome. Neurol Clin 1996;14:629-50.

29. Michaud M, Paquet J, Lavigne G, Desautels A, Montplaisir J. Sleep laboratory diagnosis of restless legs syndrome. Eur Neurol 2002;48:108-13.

30. Pizza F, Tartarotti S, Poryazova R, Baumann CR, Bassetti CL. Sleep-disordered breathing and periodic limb movements in narcolepsy with cataplexy: a systematic analysis of 35 consecutive patients. Eur Neurol 2013; 70:22-6.

31. Roux FJ. Restless legs syndrome: impact on sleep-related breathing disorders. Respirology 2013;18:238-45.

32. Sasai T, Inoue Y, Matsuura M. Clinical significance of periodic leg movements during sleep in rapid eye movement sleep behavior disorder. $J$ Neurol 2011;258:1971-8.

33. Covassin N, Neikrug AB, Liu L, Corey-Bloom J, Loredo JS, Palmer BW, et al. Clinical correlates of periodic limb movements in sleep in Parkinson's disease. J Neurol Sci 2012;316:131-6.

34. Pennestri MH, Montplaisir J, Fradette L, Lavigne G, Colombo R, Lanfranchi PA. Blood pressure changes associated with periodic leg movements during sleep in healthy subjects. Sleep Med 2013;14:555-61.

35. Sforza E, Haba-Rubio J. Night-to-night variability in periodic leg movements in patients with restless legs syndrome. Sleep Med 2005;6:259-67.

36. Haba-Rubio J, Sforza E. Test-to-test variability in motor activity during the suggested immobilization test in restless legs patients. Sleep Med 2006; 7:561-6. 